\title{
Pheochromocytoma Presenting as Acute Myocarditis with Cardiogenic Shock in Two Cases
}

\author{
Xue-Ming $\mathrm{Wu}^{1,2}$, Jien-Jiun Chen ${ }^{3}$, Cho-Kai $\mathrm{Wu}^{3}$, Lian-Yu Lin $^{1}{ }^{\text {and Chuen-Den Tseng }}{ }^{1}$
}

\begin{abstract}
Pheochromocytoma is a rare, catecholamine-secreting tumor. The classic symptoms are headache, diaphoresis, and tachycardia with paroxysmal hypertension. Other less common cardio-vascular manifestations, such as arrhythmias, angina pectoris, acute myocardial infarction, dilated cardiomyopathy, acute heart failure, and cardiogenic shock, have occasionally been reported. Here, we report two middle-aged men with acute myocarditis and cardiogenic shock, who needed an intra-aortic balloon pump and extra-corporeal membrane oxygenation for life support. They were diagnosed with pheochromocytoma and underwent laparoscopic adrenectomy that restored cardiac function. These cases illustrate diagnostic and management considerations in pheochromocytoma complicated by acute myocarditis and cardiogenic shock.
\end{abstract}

Key words: pheochromocytoma, acute myocarditis, cardiogenic shock

(Inter Med 47: 2151-2155, 2008)

(DOI: 10.2169/internalmedicine.47.1360)

\section{Introduction}

Pheochromocytoma is a tumor that produces catecholamines (1). It has a broad spectrum of clinical and biochemical manifestations, most of which are related to the paroxysmal circulation of excess catecholamines. Most patients (75\%) have the classic triad of headache, palpitation, and severe hypertension, while its hallmark - severe and labile hypertension-leads to its frequent consideration as a potential etiology in young hypertensive patients. Though most patients have either sustained or paroxysmal hypertension, some present with hypotension (2). Unusual presentations, including acute cardiogenic shock, pulmonary edema, and sudden death, have likewise been reported (3). Here, we present two cases of pheochromocytoma with an unusual presentation of acute myocarditis and cardiogenic shock.

\section{Case Report}

\section{Case 1}

A 42-year-old man presented to our emergency department with intermittent chest tightness and progressive dyspnea for one day. Past medical history showed only hypertension for a half year that was not medically managed. His family history did not show hypertension, diabetes mellitus or other hereditary diseases. He denied the use of illicit drugs. On physical examination, his temperature was 37.3 ${ }^{\circ} \mathrm{C}$, heart rate 134 beats/min, respiratory rate 30 breaths/min. His blood pressure was $228 / 117 \mathrm{mmHg}$ initially, which quickly declined to $87 / 58 \mathrm{mmHg}$. Head and neck examination was unremarkable. Lung examination revealed bilateral crackles. The heart sound was regular without murmur or friction rub. His extremities were cold and peripheral pulses were not palpable. Laboratory findings showed elevated serum urea nitrogen $(24.9 \mathrm{mg} / \mathrm{dL})$ and creatinine $(2.8 \mathrm{mg} / \mathrm{dL})$. Serum C-reactive protein level was $2.5 \mathrm{mg} / \mathrm{dL}$. The serum electrolyte profile was normal. Cardiac biologic markers

\footnotetext{
${ }^{1}$ Division of Cardiology, Department of Internal Medicine, National Taiwan University Hospital, Taipei, Taiwan, ${ }^{2}$ Department of Internal Medicine, Taoyuan General Hospital, Department of Health, Taoyuan, Taiwan and ${ }^{3}$ Department of Internal Medicine, National Taiwan University Hospital Yun-Lin Branch, Yun-Lin, Taiwan

Received for publication May 28, 2008; Accepted for publication July 17, 2008

Correspondence to Dr. Chuen-Den Tseng, cdtseng@ha.mc.ntu.edu.tw
} 
were allelevated: creatine kinase (CK) was 3,769 IU/L, creatine kinase myocardial-type isoenzyme (CK-MB) was $289.8 \mathrm{IU} / \mathrm{L}$, and troponine I was $66.7 \mathrm{mg} / \mathrm{L}$. Chest X-ray (CXR) revealed pulmonary edema (Fig. 1), while the electrocardiogram (ECG) revealed sinus tachycardia with STsegment elevation over lead V2. More prominent STsegment elevation was noted over leads V1-V3 in repeat

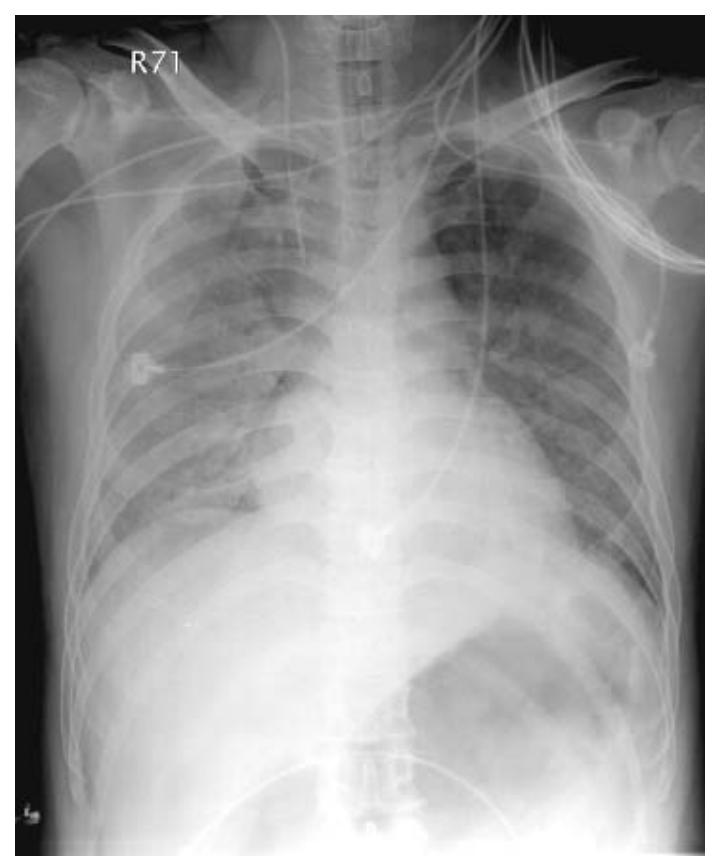

Figure 1. Chest X-ray on the day of admission showed bilateral lung edema, especially right side.
ECG one hour later (Fig. 2). Echocardiography demonstrated poor left ventricle contractility (left ventricle ejection fraction $(\mathrm{LVEF})=34 \%$ ) with severe global hypokinesia.

The patient was intubated due to hypoxic respiratory failure and intravenous inotropic agents were administered for hypotension. Emergency coronary angiography revealed patent coronary arteries. Intra-aortic balloon pump (IABP) and extra-corporeal membrane oxygenation (ECMO) support was introduced immediately. Thereafter, his hemodynamic condition improved gradually, so the IABP and ECMO support were removed on the 8th hospital day.

Figure 3 shows changes in arterial blood pressure and heart rate on the 8th hospital day. The blood pressure was quite labile and the systolic blood pressure fluctuated from $100 \mathrm{mmHg}$ to $240 \mathrm{mmHg}$. Because of the labile blood pressure, pheochromocytoma was highly suspected. We collected his 24-hour urine and the urine catecholamine levels were all elevated and confirmed by a repeat assay (Table 1). Computed tomography (CT) scan of abdomen revealed a heterogeneous mass lesion $(3.5 \times 2.7 \mathrm{~cm}$ in size $)$ over the left adrenal gland (Fig. 4). Pheochromocytoma was diagnosed and oral phenoxybenzamine was given. He underwent laparoscopic resection of the left adrenal mass on the 21th hospital day. Pathology examination confirmed pheochromocytoma with hemorrhagic transformation. The blood pressure became stable after the resection. Repeated echocardiography showed normal left ventricle function. The patient was discharged on the 32nd hospital day. He remained asymptomatic and normotensive for 13 months after discharge.

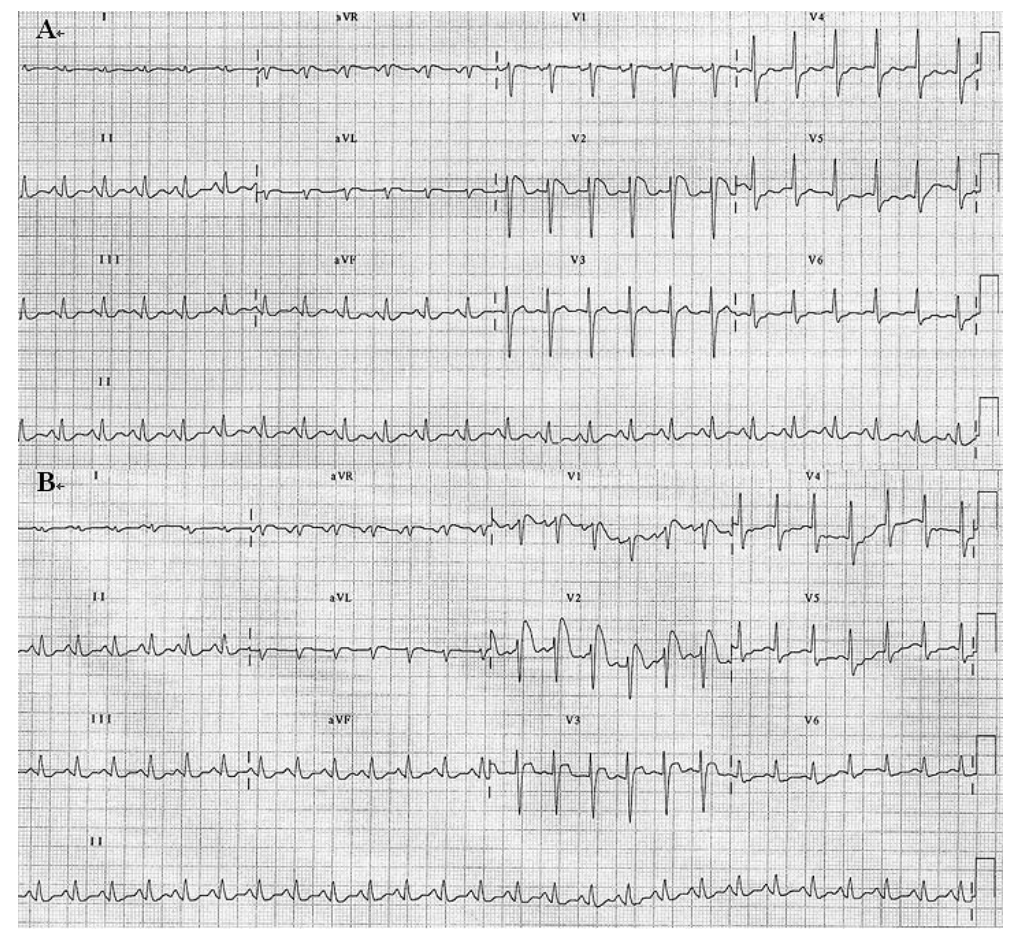

Figure 2. A: ECG on the day of admission showed sinus tachycardia with ST segment elevation over lead V2. B: ECG obtained one hour later showed sinus tachycardia with more obvious ST segment elevation over lead V1-V3. 


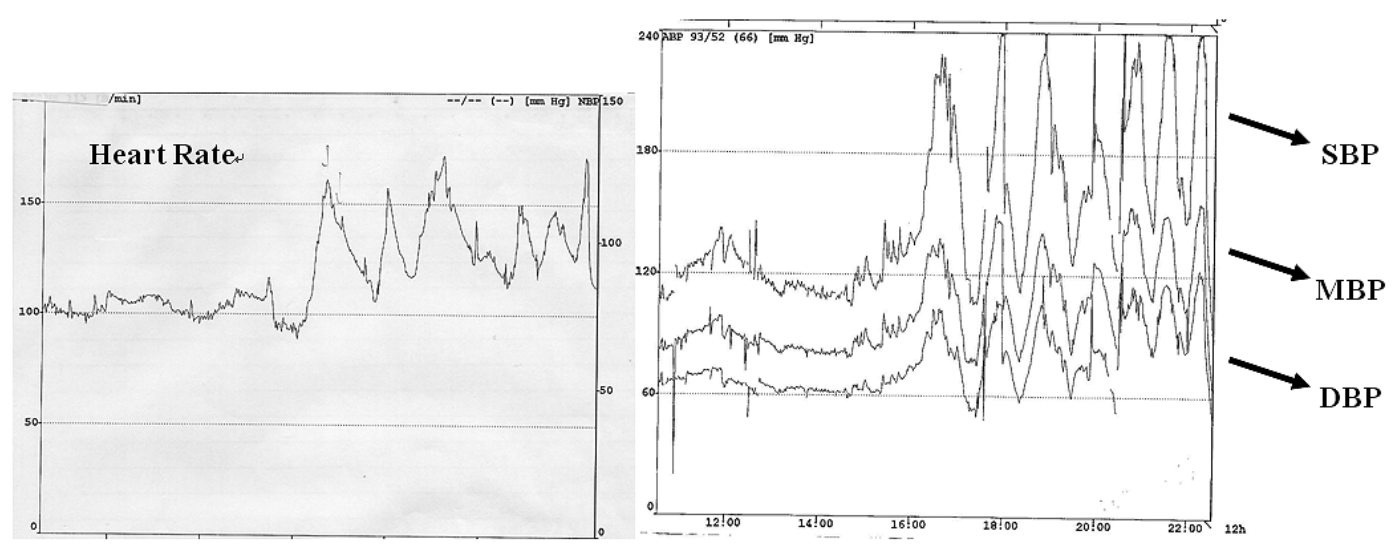

Figure 3. The labile arterial blood pressure and heart rate in case 1 obtained on the 8th hospital day (SBP: systolic blood pressure; MBP: mean blood pressure; DBP: diastolic blood pressure).

Table 1. Urine Catecholamime Levels

\begin{tabular}{l|c|c|c|c}
\hline & \multicolumn{2}{|c|}{ Case 1 } & Case 2 & \\
\hline & $4^{\text {th }}$ hospital day & $18^{\text {th }}$ hospital day & $4^{\text {th }}$ hospital day & Norm al values \\
\hline Dopamine $(\mu \mathrm{g} / 24 \mathrm{~h})$ & 6190 & 499 & 498 & $50 \sim 450$ \\
Epinephrine $(\mu \mathrm{g} / 24 \mathrm{~h})$ & 1786 & 336 & $<3$ & $<22.4$ \\
Norepinephrine $(\mu \mathrm{g} / 24 \mathrm{~h})$ & 858 & 246 & 216 & $12.1 \sim 85.5$ \\
VMA*(mg/24h) & 20.7 & 16.9 & 64.37 & $1 \sim 7$ \\
\hline
\end{tabular}

*VMA: vanillylm andelic acid
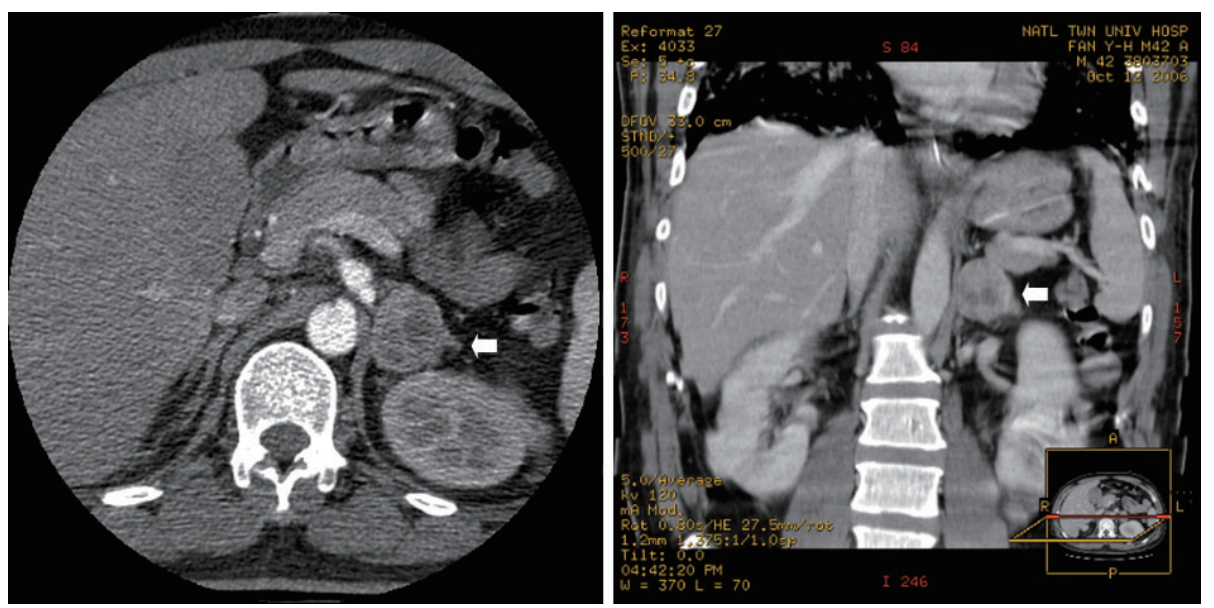

Figure 4. Abdominal CT scan of case 1 revealed a left adrenal tumor with heterogeneous soft tissue density shadow (white arrow).

\section{Case 2}

A 40-year-old man presented to our emergency department with intermittent chest tightness and dyspnea for two days. Past medical history only showed hypertension for two years that was not medically managed and his family history did not show hypertension or other systemic diseases. On arrival, his temperature was $37.5^{\circ} \mathrm{C}$ with heart rate 156 beats/min, and respiratory rate 26 breaths $/ \mathrm{min}$. His blood pressure was $212 / 110 \mathrm{mmHg}$ initially, which quickly declined to $89 / 56 \mathrm{mmHg}$. Head and neck examination was unremarkable. The lung examination revealed bilateral crackles. The heart sound was regular without murmur or friction rub. His extremities were cold and peripheral pulses were not palpable. Laboratory findings showed elevated hemoglobin $(18.5 \mathrm{~g} / \mathrm{dL})$, hematocrit $(52.5 \%)$, serum creatinine $(2.2$ $\mathrm{mg} / \mathrm{dL})$, and C-reactive protein $(2.15 \mathrm{mg} / \mathrm{dL})$. The serum electrolyte profile was moral. Elevated cardiac biologic markers were also noted (CK: 474 IU/L, CK-MB: 77.6 IU/ $\mathrm{L}$, and troponine I: $7.12 \mathrm{mg} / \mathrm{L}$ ). ECG revealed sinus tachycardia with poor $\mathrm{R}$ wave progression over precordial leads. CXR disclosed pulmonary edema with bilateral pleural effusion. Echocardiography demonstrated poor left ventricle contractility $(\mathrm{LVEF}=30 \%)$ with severe global hypokinesia and minimal amount pericardial effusion.

For severe respiratory distress and hypoxemia, he received 


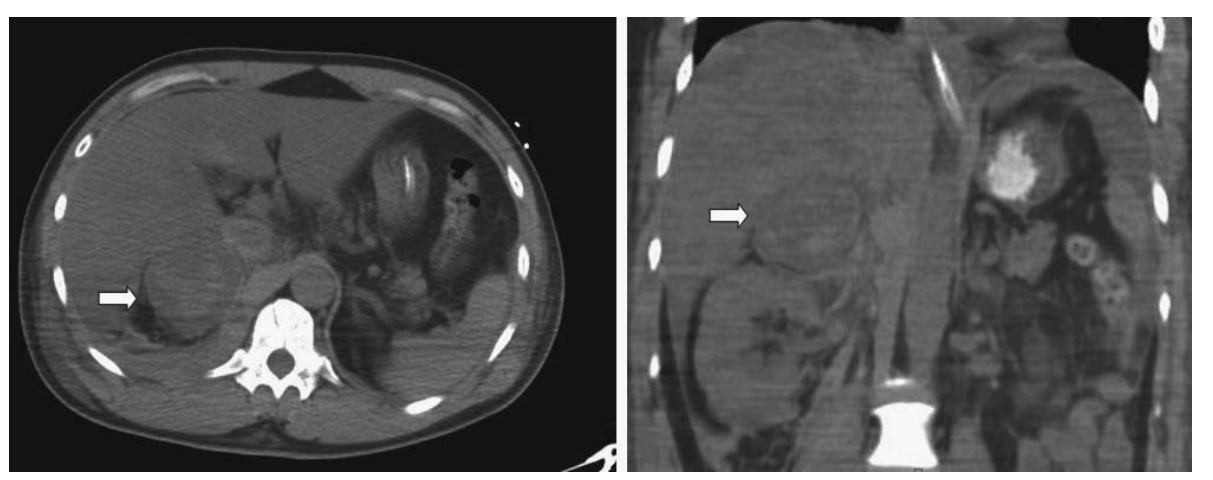

Figure 5. Abdominal CT scan of case 2 revealed a right adrenal tumor with internal mild heterogeneous density (white arrow).

endotracheal tube insertion with mechanical ventilator support. Emergency coronary angiography revealed patent coronary arteries. IABP was introduced and ECMO support was given to the patient under the diagnosis of fulminate myocarditis with cardiogenic shock. On the 2nd hospital day, his blood pressure was quite labile and the systolic blood pressure fluctuated from $100 \mathrm{mmHg}$ to $200 \mathrm{mmHg}$. We removed the IABP and ECMO support under stable hemodynamic condition on the 4th hospital day. Repeat echocardiography showed improved contractility $(\mathrm{LVEF}=67 \%)$.

Reviewing his past history, he had episodic hypertension up to $200 \mathrm{mmHg}$ with sweating and headache in recent two years. Hence, pheochromocytoma was highly suspected. The 24-hour urine catecholamine levels were all elevated (Table 1). The abdominal CT scan revealed a heterogeneous tumor (6 $\mathrm{cm}$ in size) over the right adrenal gland (Fig. 5). Based on these findings, pheochromocytoma was diagnosed and oral phenoxybenzamine was given. He underwent laparoscopic resection of the left adrenal mass on the 12th hospital day. An ovoid, yellowish and $6 \times 5 \mathrm{~cm}$ in size adrenal tumor with hemorrhage was removed. Pathology revealed pheochromocytoma with tissue necrosis and hemorrhage. In addition, left calf ischemia with gangrenous change developed during hospitalization. Therefore, he received below the knee amputation on the 12th hospital day. The patient was discharged on the 74th hospital day and remains asymptomatic for 12 months after discharge.

\section{Discussion}

These two cases demonstrate an unusual presentation of pheochromocytoma as acute myocarditis and cardiogenic shock. Although most clinicians recognize the classic symptoms of pheochromocytoma (i.e. headache, palpitation, and hypertension), diagnosis appears to be more difficult when less common symptoms occur. Unusual presentations have been reported, including dilated cardiomyopathy, acute myocarditis (4), cardiogenic shock, and sudden death. Previous case reports of catecholamine-induced myocardial injury or stunning have shown that the cardiac dysfunction can recover soon (5). The present cases also demonstrate rapid recovery of cardiac function. They had poor myocardial con- tractility initially, but were weaned off mechanical circulatory support on the 8th and 4th day, respectively.

Pheochromocytoma can be associated with hypotension and fluctuating blood pressure (6) but the frequency of presenting as shock is low (2\%) (7). Protracted hypotension leading to the so-called adrenergic shock is rare. Most pheochormocytomas secrete norepinephrine as a primary catecholamine. The etiology of hypotension is believed to be associated with excess epinephrine secretion (8). Ueda and colleagues (2) stated the high ratio of plasma epinephrine/norepinephrine indicates that epinephrine-induced vasodilatation may be mainly responsible for hypotension. However, patients with hypotension induced by predominantly norepinephrine-secreting pheochromocytomas have also been reported $(9,10)$. Excess norepinephrine can induce myocardial injury and desensitization of the blood vessels to adrenergic stimulation, which all result in cardiogenic shock. Our first case had both high daily urine epinephrine and norepinephrine level. The second case had high daily urine norepinephrine level only. Though the two cases had a different proportion of each catecholamine in secretion, the clinical presentation was similar.

The pathophysiology of shock due to pheochromocytoma is multi-factorial. Intra-vascular hypovolemia and decreased cardiac output seem to be key features of the complex pathophysiology (11). Excessive catecholamines may induce myocardial injury and some pathology studies have shown diffused interstitial inflammatory infiltrates with prevalence of lympho-monocytes and myocardial necrosis (10). Electron-microscope shows hypercontracted sacromeres with swollen mitochondria and endoplasmic reticulum (12). Because of unstable condition, we did not perform endomyocardial biopsy in these two cases. But the diagnosis of acute myocarditis could be made by clinical presentation. Due to the rapidly recovery of cardiac function, viral myocarditis is not likely. According to previous case reports, necrosis and/ or hemorrhage is frequently found within the tumor. Mohamed et al (13) stated that acute hemorrhagic necrosis of tumor may result in massive catecholamine release, as a trigger of cardiogenic shock. The adrenal tumor hemorrhage was also noticeable both grossly and microscopically in the present cases. 
Early diagnosis is difficult when physicians encounter unusual presentations of pheochromocytoma. Pheochromocytoma should be considered in patients with rapid fluctuation of the arterial blood pressure (14) and severe cardiogenic shock without coronary artery disease. In this case, the diagnosis of pheochromocytoma was made because of fluctuating blood pressure and rapidly-improved cardiac function. When pheochromocytoma is suspected, urine and serum catecholamine levels should be measured. Imaging studies, like CT scan or magnetic resonance imaging (MRI), should also be performed. MRI can disclose the tissueheterogeneity of tumor and predict intratumor hemorrhagic necrosis. In patients with unstable hemodynamic condition, bedside abdominal ultrasonography can be considered first.

Initial treatment is aimed at best hemodynamic support to prevent multi-organ failure. Intensive intra-vascular volume resuscitation is a key step in the initial treatment. The use of a mechanical circulatory device, like IABP or ECMO, is considered in the patients with poor heart function and hemodynamic condition. In those with fluctuating blood pressure, $\alpha$-blocking agents like intravenous phentolamine or oral phenoxybenzamine can be given. The timing of surgery is controversial. It is believed that pre-operative preparation with $\alpha$-adrenergic receptor blocking agents is mandatory to minimize surgical morbidity and mortality due to sudden hemodynamic changes. However, emergency surgery in pheochromocytoma may not be inferior to elective surgery under life-threatening circumstances (15).

\section{Conclusions}

Pheochromocytoma is a great imitator disease. Its recognition requires a high index of suspicion as its clinical presentation may mimic severe sepsis, acute viral myocarditis, or acute myocardial infarction. The present cases demonstrated the diagnosis of pheochromocytoma, based on the patient's history and clinical presentation. Patients with unrecognized pheochromocytoma presenting with shock have a poor prognosis. Only early recognition and careful intensive care can improve outcome.

\section{References}

1. Pacak K, Linehan WM, Eisenhofer G, Walther MM, Goldstein DS. Recent advances in genetics, diagnosis, localization, and treatment of pheochromocytoma. Ann Intern Med 134: 315-329, 2001.

2. Ueda T, Oka N, Matsumoto A, et al. Pheochromocytoma presenting as recurrent hypotension and syncope. Intern Med 44: 222227, 2005.

3. Imperadore F, Azzolini M, Piscioli F, Pusiol T, Capitanio A, Vergara G. A rare cause of cardiogenic shock: catecholamine cardiomyopathy of pheochromocytoma. Ital Heart J 3: 375-378, 2002.

4. Magalhães LC, Darze ES, Ximenes A, Santana O, Bastos J, Guimarães A. Acute myocarditis secondary to pheochromocytoma. Arq Bras Cardiol 83: 346-348; 343-345, 2004.

5. Wu GY, Doshi AA, Haas GJ. Pheochromocytoma induced cardiogenic shock with rapid recovery of ventricular function. Eur J Heart Fail 9: 212-214, 2006.

6. Magnano AR, Bai D, Bloomfield DM. Images in cardiovascular medicine. Cyclic tachycardia and hypotension. Circulation 106: e 1-e2, 2002.

7. Bergland BE. Pheochromocytoma presenting as shock. Am J Emerg Med 7: 44-48, 1989.

8. Baxter MA, Hunter P, Thompson GR, London DR. Pheaochromocytomas as a cause of hypotension. Clin Endocrinol 37: 304-306, 1992.

9. Aronoff SL, Passamani E, Borowsky BA, Weiss AN, Roberts R,
Cryer PE. Norepinephrine and epinephrine secretion from a clinically epinephrine-secreting pheochromocytoma. Am J Med 69: 321-324, 1980.

10. Baratella MC, Menti L, Angelini A, Daliento L. An unusual case of myocarditis. Int J Cardiol 65: 305-310, 1998.

11. Schifferdecker B, Kodali D, Hausner E, Aragam J. Adrenergic shock-an overlooked clinical entity? Cardiol Rev 13: 69-72, 2005.

12. Imperato-McGinley J, Gautier T, Ehlers K, Zullo MA, Goldstein DS, Vaughan ED Jr. Reversibility of catecholamine-induced dilated cardiomyopathy in a child with a pheochromocytoma. N Engl J Med 316: 793-797, 1987.

13. Mohamed HA, Aldakar MO, Habib N. Cardiogenic shock due to acute hemorrhagic necrosis of a pheochromocytoma: a case report and review of the literature. Can J Cardiol 19: 573-576, 2003.

14. Guzik P, Wykretowicz A, Wesseling IK, Wysocki H. Adrenal pheochromocytoma associated with dramatic cyclic hemodynamic fluctuations. Int J Cardiol 103: 351-353, 2005.

15. Bos JC, Toorians AW, van Mourik JC, van Schijndel RJ. Emergency resection of an extra-adrenal phaeochromocytoma: wrong or right? A case report and a review of literature. Neth J Med 61: 258-265, 2003.

(C) 2008 The Japanese Society of Internal Medicine http://www.naika.or.jp/imindex.html 